\title{
Candidate Molecules as Tumor Suppressor for Human Uterine Mesenchymal Tumor
}

\author{
Takuma Hayashi ${ }^{1,5,6}$, Akiko Horiuchi ${ }^{2}$, Susumu Tonegawa ${ }^{3}$, Ikuo Konishi ${ }^{4}$ \\ ${ }^{I}$ Dept. of Immunology and Infectious Disease, Shinshu University Graduate School of Medicine, \\ Nagano 390-8621, Japan \\ ${ }^{2}$ Horiuchi Ladies Clinic, Nagano 390-0821 Japan, \\ ${ }^{3}$ Picower Institution, Massachusetts Institute of Technology, MA 02139-4307 USA, \\ ${ }^{4}$ Dept. of Obstetrics and Gynecology, Kyoto University Graduate School of Medicine, Kyoto 606-8507, \\ Japan, \\ ${ }^{5}$ Promoting Business using Advanced Technology, Japan Science and Technology Agency (JST), Tokyo \\ 102-8666, Japan, ${ }^{6}$ SIGMA-Aldrich Collaboration Laboratory.
}

\begin{abstract}
Uterine leiomyosarcoma (Ut-LMS) develops more often in myometrium of the uterine body than in the uterine cervix. The development of gynecologic tumors is often correlated with female hormone secretion; however, the development of Ut-LMS is not substantially correlated with hormonal conditions, and the risk factor(s) are not yet known. Importantly, a diagnostic-biomarker, which distinguishes malignant tumor Ut-LMS from benign tumor leiomyoma (LMA), is yet to be established. Accordingly, it is necessary to examine risk factor(s) associated with Ut-LMS, to establish a diagnosis and a clinical treatment method. The mice with a homozygous deficiency for proteasome $\beta$-ring subunit, low-molecular mass polypeptide (LMP) $2 / \beta 1 \mathrm{i}$ spontaneously develop Ut-LMS, with a disease prevalence of $\sim 37 \%$ by 12 months of age. In the recent study, we found LMP2/ $\beta 1 \mathrm{i}$ expression to be absent in human Ut-LMS, but clearly present in other human uterine mesenchymal tumors including uterine LMA. Further analyses with clinical materials and the gene-modified mice have not clarified the biological significance of the TP53 and retinoblastoma $(\mathrm{Rb})$ pathway in malignant myometrium transformation, thus implicating LMP2/ $\beta 1 \mathrm{i}$ as an anti-tumorigenic candidate. This role of LMP2/ $\square 1 \mathrm{i}$ as a tumor suppressor may lead to new therapeutic targets in human UtLMS. (191 words)
\end{abstract}

KEYWORDS: LMP2/ $/ 1 \mathrm{i}, \mathrm{p} 53, \mathrm{Rb}$, tumor suppressor, leiomyosarcoma, mesenchymal tumor,

\section{INTRODUCTION}

The uterus is made up of three special layered linings of tissue and muscle. The middle layer of the uterus is called the myometrium, and it is also known as the muscular uterine layer. It comprises of smooth muscles which is vital during childbirth to move the baby out of the womb. Uterine mesenchymal tumors, which develop in the myometrium, have been traditionally divided into benign leiomyoma (LMA) and malignant uterine leiomyosarcoma (Ut-LMS) based on cytological atypia, mitotic activity and other criteria. Ut-LMS is relatively rare mesenchymal tumor, having an estimated annual incidence of 0.64 per 100,000 women [1]. UtLMS accounts for $2 \%$ 5\% of tumors of the uterine body and develops more often in the muscle layer of the uterine body than in the uterine cervix. As human Ut-LMS is resistant to chemotherapy and radiotherapy, surgical intervention is virtually the only means of treatment [2-4]. Ut-LMS is an aggressive malignancy, with a 5 -year survival of only $35 \%$ for tumors confined to the uterus [5]. However, developing an efficient adjuvant therapy is expected to improve the prognosis for human Ut-LMS. Uterine LMA may occur in as many as $70 \%$ 80\% of women by the age of 50 years [6]. Distinguishing human uterine LMA from Ut-LMS is very difficult, and a diagnosis generally requires surgery and cytoscopy [7]. Diagnostic categories for uterine mesenchymal tumors and morphological criteria are used to assign cases [8,9]. The non standard subtypes of uterine mesenchymal tumors such as the epithelioid and myxoid types are classified in a different way using these features, so the establishment of a diagnostic method for the identification of non-standard smooth muscle differentiation is important $[8,9]$.

High estrogen levels are considered to significantly influence the development of tumors in the uterine body [10-12]. The molecular mechanisms by which uterine LMA and human Ut-LMS transform are not yet known, though tumors that have initiated and grown in the myometrium for some reason gradually become larger due to the influence of the female hormone, estrogen, and generate tumors.

However, no correlation between the development of human Ut-LMS and hormonal conditions, and no 
obvious risk factors, have been found. Although cases accompanied by hypocalcaemia or eosinophilia have been reported, neither clinical abnormality is an initial risk factor for human Ut-LMS. The TP53 tumor suppressor pathway is one of the most well characterized pathways in various malignant tumors. The retinoblastoma $(\mathrm{Rb})$ pathway furthermore represents a second major tumor suppressor pathway deregulated in many malignant tumors. However, correlation between these pathways and human Ut-LMS tumorigenesis is unclearly understood unlike epithelial malignant tumors i.e. cancer.The ubiqutin-proteasomal degradation pathway is essential for many cellular processes, including the cell cycle and the regulation of gene expression. In structure, the proteasome is a cylindrical complex containing a core of four stacked rings around a central pore, each ring composed of seven individual proteins. The inner two rings are made of seven $\beta$ ring subunits that contain three to seven protease active sites. Alternative $\beta$-ring forms denoted LMP2/ $\beta 1 \mathrm{i}$ can be expressed in the myometrium in response to exposure to pro-inflammatory signals such as cytokines, in particular, interferon (IFN)- $\gamma$. Ut-LMS reportedly occurred in female LMP2/ $\beta 1$ i-deficient mice at age 6 months or older, and the incidence at 12 months of age was about $37 \%$. The determination of the malignant potential of mesenchymal tumors also represents a significant diagnostic conundrum with important therapeutic ramifications. However, the genetic changes underlying the neoplastic transformation of uterine smooth muscle cells have not been fully characterized. The identification of a risk factor and/or biological candidate(s) associated with the development of human Ut-LMS, i.e. LMP2/ $\beta 1$ i, would significantly contribute to the development of preventive and therapeutic treatments.

\section{DEVELOPMENT OF UT-LMS IN LMP2/ß1I-DEFFICIENT MICE.}

Cytoplasmic proteins are mostly degraded by a protease complex, which has many substrates consisting of twenty-eight 20 to $30-\mathrm{kDa}$ subunits, referred to as the $20 \mathrm{~S}$ proteasome, and it is located in the nucleus and the cytoplasm [13,14]. The ubiqutin-proteasome degradation pathway is essential for many cellular processes, including the cell cycle, the regulation of gene expression and immunological function [15]. IFN- $\gamma$ induces the expression of large numbers of responsive genes, proteasome subunits, i.e., LMP2/ $\beta 1 \mathrm{i}$, LMP7/ $/ 35 \mathrm{i}$

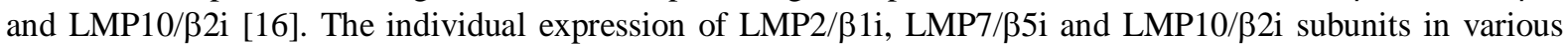
cell types or tissues is believed to contribute to the initiation and development of disorders. A recent study revealed a unique role for LMP7/ $/ 35 \mathrm{i}$ in controlling pathogenic immune responses and provided a therapeutic rationale for targeting LMP7/ $\beta 5 \mathrm{i}$ in autoimmune disorders, especially rheumatoid arthritis [17].

Recent reports demonstrate LMP2/ $\beta 1$ i as obligatory for tumor surveillance and a tissue- specific role for LMP2/ $\beta 1 \mathrm{i}$ in protection from spontaneous uterus neoplasms $[18,19]$. Homozygous mice deficient in LMP2/ $/ 1$ 1i show tissue- and substrate- dependent abnormalities in the biological functions of the proteasome $[18,20]$. Ut-LMS reportedly occurred in female LMP2/ $\beta 1$ i-deficient mice at age 6 months or older, and the incidence at 14 months of age was about $40 \%[19,20]$ (Figure 1). The disease prevalence in mice is similar to that of human Ut-LMS, which occurs after menopause [19]. Pathological studies of LMP2/31i-deficient uterine tumors have revealed characteristic abnormalities of human Ut-LMS [19]. The tumors lacked lymphoid infiltrates, a sign of immune recognition, and consisted of uniform elongated myometrium cells arranged into bundles (Figure 1). The nuclei of the tumor cells varied in size and shape, furthermore, mitosis was frequent. The tumor consisted of uniform elongated myometrium cells arranged into bundles. In contrast, the myometrium cells of its parental mice, C57BL/6 mice were normal in appearance $[19,20]$. Whereas relatively few MIB1/ki-67-positive cells, the proliferating cells, were observed in the basal cell layer of the normal myometrium, most of the basal cells in LMP2/ $\beta 1$ i-deficient mice vividly expressed MIB1/ki-67 [19] (Figure 1). This immunohistochemistry (IHC) study indicates abnormal proliferation of the LMP2/ $\beta 1$ i-lacking cells in the basal layer. LMP2/ß1i-deficient mice that have developed Ut-LMS undergo considerable body-weight loss, and then die by 14 months of age. They also potentially exhibit skeletal muscle metastasis from the Ut-LMS [21]. Therefore these research findings suggest that LMP2/B1i-deficient mice with Ut-LMS die as a result of tumor growth and metastasis. In general, it is not easy to distinguish uterine mesenchymal tumors from human UtLMS, however, in mice, because of such characteristic pathological findings, significant body-weight loss, and skeletal muscle metastasis, a tumor that develops in the uterus of an LMP2/ $\beta 1$ 1i-deficient mouse can be considered malignant, i.e., an Ut-LMS [19-21].

\section{DEFECTIVE LMP2/B1I EXPRESSION IN HUMAN UT-LMS}

The non-standard subtypes of uterine mesenchymal tumors such as the epithelioid and myxoid types are classified in a different way using these features, so the establishment of a diagnostic method for the identification of non-standard smooth muscle differentiation is important [7-9]. IHC studies were performed to demonstrate the validity and reliability of LMP2/ $\beta 1 \mathrm{i}$ as a diagnostic biomarker under the combination of other candidate molecules, for instance cyclin $\mathrm{E}$ and calponin $\mathrm{h} 1$ [22,23].

Of the 54 cases we examined with human Ut-LMS, 46 cases were negative for LMP2/ $\beta 1$ i expression, 4 
cases were focally positive, and 2 cases were partially positive [23]. Two human Ut-LMS cases were stained for LMP2/ $\beta 1$ i. The expression levels of LMP2/ $\beta 1 \mathrm{i}$ were also evaluated in skeletal muscle and rectum metastases from individual human Ut-LMS patients [23]. Histological findings were consistent with metastatic LMS for the skeletal muscle and rectum lesions. In western blotting and RT-PCR experiments, LMP2/ $\beta 1 \mathrm{i}$ was expressed in normal myometrium, but not in human Ut-LMS, both strongly supportive of the IHC findings [22-24] (Figure 2).

\section{TUMOR SUPPRESSOR AND ONCOGENIC PATHWAY IN UT-LMS}

The TP53 pathway: Molecular analyses have shown that many of the canonical tumor suppressor pathways, such as the TP53 and retinoblastoma ( $\mathrm{Rb})$ pathways are ablated in these tumors [25]. Furthermore, some sarcomas also harbor activating oncogenic mutations; such as expression of oncogenic K-ras. Together, disruption of these genes and pathways are thought to be a driving force in sarcomagenesis. The TP53 tumor suppressor pathway is one of the most well characterized pathways in cancers [26]. The TP53 gene encodes a transcription factor required for the activation of numerous DNA damage-dependent checkpoint response and apoptotic genes [27,28], and thus its activities are often ablated in many cancers. In addition to loss of TP53 functions via inherited germline somatic mutations, the TP53 pathway is commonly disrupted by point mutations in the TP53 gene during sporadic sarcomagenesis [29]. However, even though TP53 gene alterations are widely regarded as having a significant impact on sarcomagenesis, many sarcomas retain wild type TP53, yet phenotypically display a loss of TP53 function. These findings suggest that changes in other components of the TP53 pathway; such as amplification of Mdm2, a negative regulator of the TP53 pathway, may result in TP53 inactivation [30,31]. Furthermore, both mice and humans with elevated levels of Mdm2 due to a high frequency single nucleotide polymorphism in the Mdm2 promoter (Mdm2SNP309) are more susceptible to sarcoma formation [32-34]. Additionally, deletion or silencing of $\mathrm{p} 19^{A r f}$ (p14Arf in human), an inhibitor of the MDM2-TP53 axis, often results in development of sarcomas. Together, these data indicate that while inactivation of the TP53 pathway is observed in the vast majority of human sarcomas, the mechanisms leading to disruption of the pathway can vary greatly.

People who inherit only one functional copy of the TP53 gene will most likely develop tumors in early adulthood, a disease known as Li-Fraumeni syndrome. More than 50 percent of human tumors contain a mutation or deletion of the TP53 gene [35]. To increase tumor incidence and better assess the role of systemic expression of p53 in responses to initiation of Ut-LMS tumorigenesis, LMP2/ $\beta 1$ i-deficient mice were bred with Tp53-deficient mice to create $\operatorname{Lmp} 2^{-1-} T p 53^{-/-}$double knockout mice. Ut-LMS incidence and death rates were similar in $\operatorname{Lmp} 2^{-/} T p 53^{-/-}$mice and closely matched those for control $L m p 2^{-/-} T p 53^{+/+}$mice. The correlation between defective p53 function and Ut-LMS tumorigenesis is unclear. Although we previously demonstrated that the abnormal expression of ovarian steroid receptors, p53 and MIB1/ki-67 and mutations of p53 were frequently associated with human Ut-LMS, defective LMP2/ $\beta 1$ i expression appears to be more characteristic of Ut-LMS than any of these factors [22-24].

The retinoblastoma (Rb) pathway: The Rb pathway represents a second major tumor suppressor pathway deregulated in many sarcomas. Individuals inheriting a germline $R b$ mutation typically develop cancers of the eye early in life [36-38]. However, in addition to retinal cancers, these children have a significantly higher propensity to develop sarcomas than the general population [39]. While inheritance of a germline $R b$ alterations increases sarcoma risk, there are also numerous examples of sporadic sarcomas harboring spontaneous $\mathrm{Rb}$ somatic mutations and deletions, particularly osteosarcomas and rhabdomyosarcomas [40]. Furthermore, p16 ${ }^{l k n t a}$, a negative regulator of the CDK-cyclin complexes that phosphorylate and activate $\mathrm{Rb}$, is often deleted in sarcomas $[41,42]$.

Long term survivors of hereditary retinoblastoma are at risk of developing a variety of non-ocular second primary malignancies, the most common histological subtype of which is osteosarcoma. The reported risk varies widely, but a cumulative incidence of $1 \%$ for each year of life has been suggested as an approximate estimate. Earlier reports indicated that many of these malignancies are radiation or chemotherapy induced [4346]. However, more recent studies [46-49] suggest that the development of second primary neoplasms in patients with hereditary retinoblastoma results partly from a genetic predisposition and partly from the potentiating effect of radiotherapy on tumorigenesis through mutation of the second $R b$ gene (RB1) allele, the first $R B 1$ allele being mutated in all patients with hereditary retinoblastoma [50]. A recent analysis of second primary neoplasms in retinoblastoma survivors revealed that $76 \%$ of the second tumors occurred in the head and neck region and that soft tissue sarcomas

were the single largest category, comprising $24 \%$ of the total [49]. The median age at diagnosis of a second tumor for the entire series was 16.4 years. The youngest patient to develop a soft tissue tumor was 4 
years old and the oldest was 41. In previously reported cases of leiomyosarcoma occurring outside the head and neck region, the patients have been in the 4th to 6th decades. Importantly, in female patients, uterine leiomyosarcoma has not been reported after hereditary $\mathrm{Rb}$, although simultaneously occurring benign Ut-LMS have been described [51].

Recent reports describe three patients who developed leiomyosarcomas, one involving the subcutaneous tissue of the thigh and the pelvic soft tissues and the other the urinary bladder, following hereditary retinoblastoma 36,38 and 49 years earlier, respectively [51,52]. We also have reported a case of primary bladder LMS in a 45-year-old woman with a history of hereditary Rb. The cases we report, together with others described, suggest that with increasing duration of survival after hereditary retinoblastoma there is an increased risk of developing leiomyosarcoma in areas remote from the site of irradiation. Such patients thus require appropriate follow up. Over all research experiments including with gene-deficient mouse models and clinical research suggest that defective Rb expression does not take part in Ut-LMS onset.

\section{CONCLUSION}

In the case of gynecological cancers, such as breast cancer, a female hormonal imbalance is often a risk factor for developing tumors [10-12]. As in the case of uterine LMA, however, a correlation between the development of human Ut-LMS, the female hormone, and hormone receptors has been unclear. A recent report showed the expression of $L M P 2 / \beta 1 i$ mRNA and protein in luminal and glandular epitheliua, placenta villi, trophoblastic shells, and arterial endothelial cells [53-55]. These results implicate LMP2/ $\beta 1 \mathrm{i}$ in the invasion of placental villi, degradation of the extracellular matrix, immune tolerance, glandular secretion, and angiogenesis [53-55]. However, these findings do not help to elucidate the regulatory role of LMP2/ $\beta 1 i$ in human Ut-LMS tumorigenesis. The LMP2/ $/$ 1i-deficient mouse was the first animal model of spontaneous Ut-LMS to be established $[19,22,23]$. In the recent studies, LMP2/ $\beta 1 \mathrm{i}$ is reported to negatively regulate human Ut-LMS independently of its role in the proteasome [56-59]. Moreover, several lines of evidence indicate that calcium binding protein, calponin h1 clearly affects LMP2/ $\beta 1$ i-induced cellular morphological chances $[58,59]$. Further experiments are also required to elucidate the molecular mechanism of human Ut-LMS tumorigenesis involved biological significance of LMP2/ $\beta 1$ i. Histologic and IHC characteristics of uterine mesenchymal tumors including mitotically active leiomyoma, bizarre leiomyoma, lipoleiomyoma, uterine smooth muscle tumors of uncertain malignant potential (STUMP), leiomyomatoid angiomatous neuroendocrin tumor (LANT) are summarized [60-65]. Clarification of the correlation between these factors and the development of human UtLMS and the identification of specific risk factors may lead to the development of new clinical treatments for the disease.

\section{FINAL CONSIDERATIONS}

Human Ut-LMS is refractory to chemotherapy and has a poor prognosis. Defective LMP $/ \beta 1 \mathrm{i}$ expression is likely to be one of the risk factors in the development of human Ut-LMS as it is in the LMP2/ $\beta 1$ ideficient mouse. While mouse model can not completely predict the outcome of Ut-LMS, the molecular biological and cytological information obtained from LMP2/ $\beta 1$ i-deficient mice and human clinical materials will contribute remarkably to the development of preventive methods, a potential diagnostic-biomarker, and new therapeutic approaches against human mesenchymal tumors, especially human Ut-LMS.

\section{ACKNOWLEDGEMENTS:}

We sincerely thank Professor Luc Van Kaer (Vanderbilt University Medical Centre) for research support. This study was supported in part by grants from the Ministry of Education, Culture, Science and Technology, and The Foundation of Osaka Cancer Research, The Ichiro Kanehara Foundation for the Promotion of Medical Science and Medical Care, The foundation for the Promotion of Cancer Research, The Kanzawa Medical Research Foundation, The Shinshu Medical Foundation, and The Takeda Foundation for Medical Science.

\section{REFERENCES}

[1] Zaloudek C, Hendrickson MR. Mesenchymal tumors of the uterus, in Kurman RJ.(ed): Blaustein`s Pathology of the Female Genital Tract (ed 5). New York, Springer-Verlag 2002; 5: 561-78.

[2] Wu TI, Chang TC, Hsueh S, Hsu KH, Chou HH, Huang HJ, Lai CH. Prognostic factors and impact of adjuvant chemotherapy for uterine leiomyosarcoma. Gynecol. Oncol. 2006; 100: 166-72.

[3] Leitao MM, Soslow RA, Nonaka D, Olshen AB, Aghajanian C, Sabbatini P, Dupont J, Hensley M, Sonoda Y, Barakat RR, Anderson S. Tissue microarray immunohisto chemical expression of estrogen, progesterone, and androgen receptors in uterine leiomyomata and leiomyosarcoma. Cancer 2004; 101: 1455-62.

[4] Perez EA, Pusztai L, Van de Vijver M. Improving patient care through molecular diagnostics. Semin. Oncol. 2004; 31: 14-20. 
[5] http://www.cancer.gov/cancertopics/pdq/treatment/uterinesarcoma/HealthProfessional/page1\#Section_87

[6] http://cancer.gov/cancertopics/pdq/treatment/uterinesarcoma/HealthProfessional

[7] Evans HL, Chawla SP, Simpson C, Finn KP. Smooth muscle neoplasms of the uterus other than ordinary leiomyoma. A study of 46 cases, with emphasis on diagnostic criteria and prognostic factors. Cancer 1988; 62: 2239-47.

[8] Kurma RJ. Pathology of the Female Genital Tract, 4th ed. New York, Springer-Verlag; $2001 ; 4: 499$.

[9] Diagnostic Criteria for LMS, Adapted from 2003 WHO Guidelines: (2003) World Health Organization Classification of Tumours: Pathology and Genetics, Pathology and Genetics of Tumours of the Breast and Female Genital Organs. IARC Press, France.

[10] Lin JF, Slomovitz BM. Uterine sarcoma. Curr. Oncol. Rep. 2008; 10: 512-8.

[11] Amant F, Coosemans A, Debiec-Rychter M, Timmerman D, Vergote I. Clinical management of uterine sarcomas. Lancet Oncol. 2009; 10: 1188-98.

[12] Miettinen M, Fetsch JF. Evaluation of biological potential of smooth muscle tumours. Histopathol. 2006; 48: 97-105.

[13] Peters JM, Franke WW, Kleinschmidt JA. Distinct $19 \mathrm{~S}$ and $20 \mathrm{~S}$ subcomplexes of the $26 \mathrm{~S}$ proteasome and their distribution in the nucleus and the cytoplasm. J. Biol. Chem. 1994; 269: 7709-18.

[14] Lodish H, Berk A, Matsudaira P, Kaiser CA, Krieger M, Scott MP, Zipursky SL, Darnell J. 2004 "3". Mol Cell Biol (5th ed.). New York: W.H. Freeman and CO. 2004; 5: 66-72.

[15] Konstantinova IM, Tsimokha AS, Mittenberg AG. Role of proteasomes in cellular regulation. Intl. Rev. Cell. Mol. Biol. 2008; 267: 59-124.

[16] Wang J, Maldonado MA. The Ubiquitin-Proteasome System and Its Role in Inflammatory and Autoimmune Diseases. Cell. Mol. Immunol. 2006; 3: 255-61.

[17] Muchamuel T, Basler M, Aujay MA, Suzuki E, Kalim KW, Lauer C, Sylvain C, Ring ER, Shields J, Jiang J, Shwonek P, Parlati F, Demo SD, Bennett MK, Kirk CJ, Groettrup M. A selective inhibitor of the immunoproteasome subunit LMP7 blocks cytokine production and attenuates progression of experimental arthritis. Nature Med. 2009; 15: 781-8.

[18] Van Kaer L, Ashton-Rickardt PG, Eichelberger M, Gaczynska M, Nagashima K, Rock KL, Goldberg AL, Doherty PC, Tonegawa S. Altered peptidase and viral-specifi c T cell response in LMP2 mutant mice. Immunity 1994; 1: 533-41.

[19] Hayashi T, Faustman DL. Development of spontaneous uterine tumors in low molecular mass polypeptide-2 knockout mice. Cancer Res. 2002; 62: 24-7.

[20] http://www.informatics.jax.org/javawi2/servlet/WIFetch?page=alleleDetail\&id=MGI:2152729\# refs

[21] Hayashi T, Horiuchi A, Sano K, Hiraoka N, Kanai Y, Shiozawa T, Tonegawa S, Konishi I. Molecular approach on uterine leiomyosarcoma: LMP2-deficient mice as an animal model of spontaneous uterine leiomyosarcoma. Sarcoma $2011: 476498$. Epub 2011 Mar 8.

[22] Hayashi T, Kobayashi Y, Kohsaka S, Sano K. mutation in the ATP-binding region of JAK1, identified in human uterine leiomyosarcomas, results in defective interferon-gamma inducibility of TAP1 and LMP2. Oncogene 2006; 25: 4016-26.

[23] Hayashi T, Horiuchi A, Sano K, Hiraoka N, Kasai M, Ichimura T, Nagase S, Ishiko O, Kanai Y, Yaegashi N, Aburatani H, Shiozawa T, Tonegawa S, Konishi I. Potential role of LMP2 as tumor-suppressor defines new targets for uterine leiomyosarcoma therapy. Sci. Rep. 2011; 1:180| DOI:10.1038/srep00180

[24] Zhai YL, Kobayashi Y, Mori A, Orii A, Nikaido T, Konishi I, Fujii S. Expression of steroid receptors, Ki-67, and p53 in uterine leiomyosarcomas. Intl. J. Gynecol. Pathol. 2004; 18: 20-8.

[25] Toguchida J, Nakayama T: Molecular genetics of sarcomas: applications to diagnoses and therapy. Cancer Sci 2009; 100: 157380 .

[26] Vogelstein B, Lane D, Levine AJ: Surfing the p53 network. Nature 2000, 408(6810):307-310.

[27] Raycroft L, et al: Analysis of p53 mutants for transcriptional activity. Mol Cell Biol 1991; 11: 6067-74.

[28] Raycroft L, Wu HY, Lozano G: Transcriptional activation by wild-type but not transforming mutants of the p53 anti-oncogene. Science 1990; 249: 1049-51.

[29] Wang LL: Biology of osteogenic sarcoma. Cancer J 2005, 11(4):294-305.

[30] Oliner JD, et al: Amplification of a gene encoding a p53-associated protein in human sarcomas. Nature 1992; $358: 80-3$.

[31] Oliner JD, et al: Oncoprotein MDM2 conceals the activation domain of tumour suppressor p53. Nature 1993; 362: 857-60.

[32] Bond GL, et al: A Single Nucleotide Polymorphism in the MDM2 Promoter Attenuates the p53 Tumor Suppressor Pathway and Accelerates Tumor Formation in Humans. Cell 2004; 119: 591-602.

[33] Ito M, et al: Comprehensive Mapping of p53 Pathway Alterations Reveals an Apparent Role for Both SNP309 and MDM2 Amplification in Sarcomagenesis. Clin Cancer Res 2011; 17: 416-26.

[34] Post SM, et al: A High-Frequency Regulatory Polymorphism in the p53 Pathway Accelerates Tumor Development. Cancer Cell 2010; 18: 220-30.

[35] Hollstein M, Sidransky D, Vogelstein B, Harris CC. p53 mutations in human cancers Science 1991; 253: 49-53.

[36] Tebbet RD, Vickery RD: Osteogenic sarcoma following irradiation for retinoblastoma; with the report of a case. Am J Ophthalmol 1952; 35: 811-18.

[37] Nordling CO: A new theory on cancer-inducing mechanism. Br J Cancer 1953, 7(1):68-72.

[38] 34. Knudson AG Jr: Mutation and cancer: statistical study of retinoblastoma. Proc Natl Acad Sci U S A 1971; 68: 820-3.

[39] Deshpande A, Hinds PW: The retinoblastoma protein in osteoblast differentiation and osteosarcoma. Curr Mol Med 2006; 6: 809-17.

[40] Toguchida J, et al: Preferential mutation of paternally derived RB gene as the initial event in sporadic osteosarcoma. Nature 1989; 338: 156-8.

[41] Yonghao T, et al: Deletions and point mutations of p16, p15 gene in primary tumors and tumor cell lines. Chin Med Sci J 1999; 14: $200-5$.

[42] Oda Y, et al: Frequent alteration of p16(INK4a)/p14(ARF) and p53 pathways in the round cell component of myxoid/round cell liposarcoma: p53 gene alterations and reduced p14(ARF) expression both correlate with poor prognosis. J Pathol 2005; 207: 41021.

[43] Martin-Hirsch DP, Habashi S, Benbow EW, et al. Post-irradiation leiomyosarcoma of the maxilla. J Laryngol Otol 1991; 105: $1068-71$.

[44] Kawamura J, Sakurai M, Tsukamoto K, et al. Leiomyosarcoma of the bladder eighteen years after cyclophosphamide therapy for retinoblastoma. Urol Int 1993; 51: 49-53.

[45] Schwarz MB, Burgess LPA, Fee WE, et al. Post-irradiation sarcoma in retinoblastoma. Induction or predisposition? Arch Otolaryngol Head Neck Surg 1988; 114: 640-4.

[46] Moll AC, Imhof SM, Bouter LM, et al. Second primary tumours in patients with hereditary retinoblastoma: a register-based 
follow-up study, 1945-1994. Int J Cancer 1996; 67: 515-9.

Imhof SM, Moll AC, Hofman P, et al. Second primary tumours in hereditary- and nonhereditary retinoblastoma patients treated with megavoltage external beam irradiation. Doc Ophthalmol 1997; 93: 337-44.

Mohney BG, Robertson DM, Schomberg PJ, et al. Second monocular tumours in survivors of heritable retinoblastoma and prior radiation therapy. Am J Ophthalmol 1998; 126: 269-77.

Abramson DH, Melson MR, Dunkel IJ, et al. Third (fourth and fifth) nonocular tumors in survivors of retinoblastoma. Ophthalmology 2001; 108: 1868-76.

Chauvienc L, Mosseri V, Quintana E, et al. Osteosarcoma following retinoblastoma: age at onset and latency period. Ophthalmic Genet 2001; 22: 77-88.

Liang SX, Lakshmanan Y, Woda BA, et al. A high grade primary leiomyosarcoma of the bladder in a survivor of retinoblastoma. Arch Pathol Lab Med 2001; 125: 1231-4.

Venkatraman L, Goepel J R, Steele K, et al. Soft tissue, pelvic, and urinary bladder Soft tissue, pelvic, and urinary bladder hereditary retinoblastoma. J Clin Pathol 2003; 56: 233-6

Wang HX, Wang HM, Li QL, Judoson PL. Expression of proteasome subunits low molecular mass polypeptide (LMP) 2 and LMP7 in the endometrium and placenta of rhesus monkey (Macaca mulatta) during early pregnancy. Biol. Reprod. 2004; 71: 1317-24.

Wang, HX., Wang, HM., Lin, HY., Yang, Q., Zhang, H., Tsang, BK. \& Zhu, C. Proteasome subunit LMP2 is required for matrix metalloproteinase-2 and -9 expression and activities in human invasive extravillous trophoblast cell line. J. Cell. Physiol. 2006; 206: 616-23.

Fu JJ, Lin P, Lv XY, Yan XJ, Wang HX, Zhu C, Tsang BK, Yu XG, Wang H. Low molecular mass polypeptide-2 in human trophoblast: over-expression in hydatidiform moles and possible role in trophoblast cell invasion. Placenta. 2009; 30: 305-12. Hayashi T, Horiuchi A, Aburatani H, Yaegashi N, Tonegawa S, Konishi I. A potential diagnostic biomarker: Proteasome LMP2/b1i-differential expression in human uterus neoplasm . Nature Precedings 2012; March 2: hdl:10101/npre.2012.7082.1.

Horiuchi A, Nikaido T, Ito K, Zhai Y, Orii A, Taniguchi S, Toki T, Fujii S. Reduced expression of calponin h1 in leiomyosarcoma of the uterus. Lab. Invest. 1998; 78: 839-46.

Horiuchi A, Nikaido T, Taniguchi S, Fujii S. Possible role of calponin h1 as a tumor suppressor in human uterine leiomyosarcoma. J. Natl. Cancer Inst. 1999; 91: 790-6.

Hayashi T, Horiuchi A, Sano K, Hiraoka N, Kasai M, Ichimura T, Nagase S, Ishiko O, Kanai Y, Yaegashi N, Aburatani H, Shiozawa T, Tonegawa S, Konishi I. Potential role of LMP2 as an anti-oncogenic factor in human uterine leiomyosarcoma: morphological significance of calponin h1. FEBS Letter 2012; 586: 1824-31.

Zhai YL, Nikaido T, Shiozawa T, Orii A, Fujii S. Expression of cyclins and cyclin-dependent kinases in smooth muscle tumors of the uterus. Intl. J. Cancer 1999; 84: 224-50.

Ip PP, Cheung AN, Clement PB. Uterine smooth muscle tumors of uncertain malignant potential (STUMP): a clinicopathologic analysis of 16 cases. Am. J. Surg. Pathol. 2009; 33: 992-1005.

Ip PP, Tse KY, Tam KF. Uterine smooth muscle tumors other than the ordinary leiomyomas and leiomyosarcomas: a review of selected variants with emphasis on recent advances and unusual morphology that may cause concern for malignancy. Adv. Anat. Pathol. 2010; 17: 91-112.

Vajtai I, Sahli R, Kappeler A, Christ ER. Seiler RW. Leiomyomatoid angiomatous neuroendocrine tumor (LANT) of the pituitary: a distinctive biphasic neoplasm with primitive secretory phenotype and smooth muscle-rich stroma. Acta Neuropathol. 2006; 111: 278-83.

Sakashita N, Yamada M, Nakagawa T, Yamasaki H, Takeya M. A leiomyomatoid angiomatous neuroendocrine tumor of the myometrium: case study with ultrastructural analysis. Hum. Pathol. 2008; 39: 788-92.

Avritscher R, Iyer1 RB, Ro J, Whitman G. Lipoleiomyoma of the Uterus. Am. J. Radiol. 2001; 177: 856

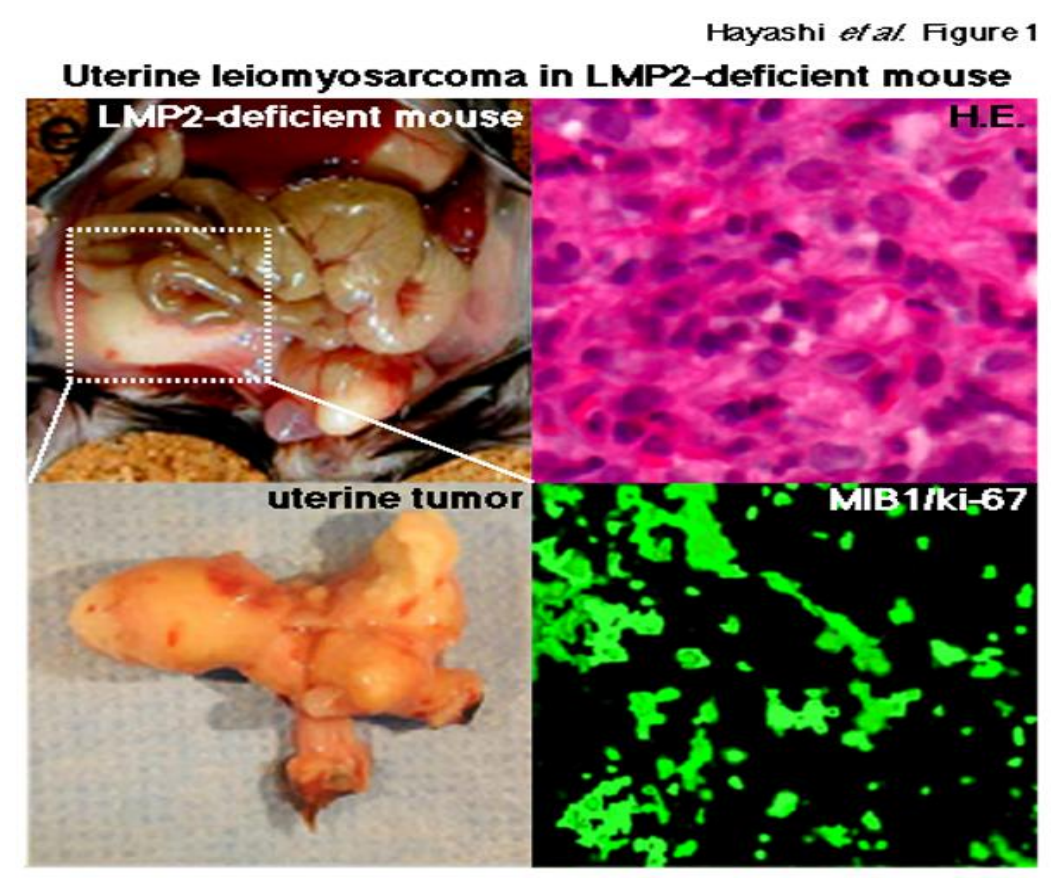

Figure 1. Homozygous mice deficient in LMP2/ $/ 11$, an interferon (IFN)- $\gamma$-inducible factor, show tissue- 
and substrate-dependent abnormalities in the biological functions of the proteasome ${ }^{19,20}$. Ut-LMS reportedly occurred in female LMP2/ $\beta 1$ i-deficient mice at age 6 months or older, and the incidence at 14 months of age was about $40 \%{ }^{19,20}$

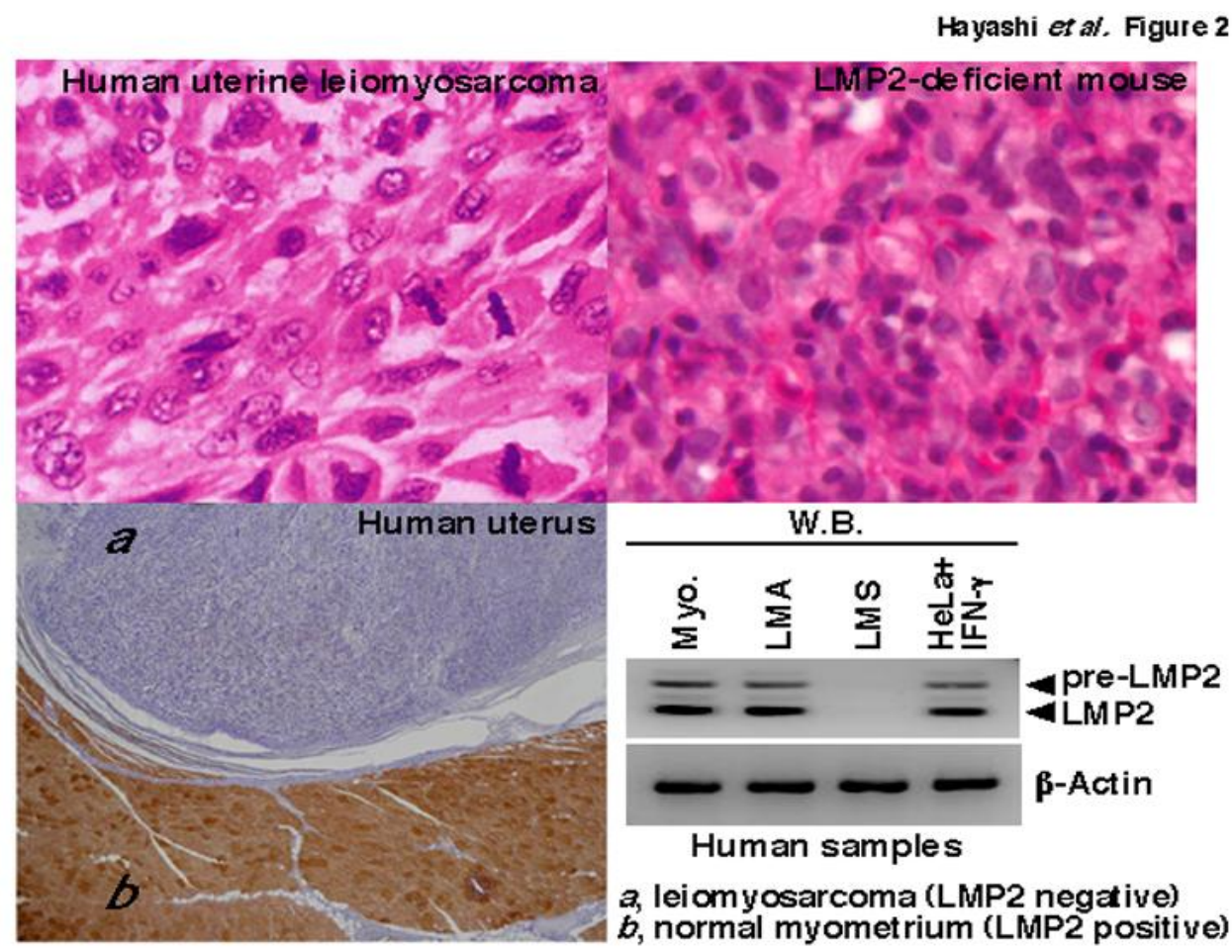

Figure 2. Defective LMP2/ $\beta$ 1i expression in human uterine leiomyosarcoma. H.E. staining tissue sections of uterine leomyosarcoma of LMP2/ $\beta 1$ i-deficient mouse and patient. Immunohistochmical staining tissue section of human uterine leiomyosarcoma with anti-human LMP2/ $\beta 1$ i monoclonal antibody. Extracts of $50 \mu$ gere resolved by $10 \%$ sodium dodecyl sulfate-polyacrylamide gel electrophoresis (SDS-PAGE). The levels of LMP2 and $\beta$-actin were examined by western blot (W.B.) analysis with appropriate antibodies. Myo.; human myometrium, LMA; human leiomyoma, LMS; human leiomyosarcoma, HeLa+IFN- $\gamma$; HeLa cells treated by IFN- $\gamma$. 